\title{
2-D Parameters Estimation Algorithm for Near-Field Sources Based on Polarization Sensitive Array
}

\author{
Yanbo Liu ${ }^{1, a}$ and Binbin $\mathrm{Yu}^{1, b^{*}}$ \\ ${ }^{1}$ Information Technology and Media College of Beihua University, Jilin 132013, China \\ a28364335@qq.coml, b22342690@qq.com
}

Key words: Polarization Sensitive Array; Near-Field; Bearing; Range

\begin{abstract}
Proposed a 2-D parameters estimation algorithm for near-field sources based on polarization sensitive array. The algorithm adopted the centro-symmetric uniform linear array, constructed three covariance matrices by the particular array labeling, and taken advantage of the total least squares and subspace decomposition method to estimate the bearing and range parameters. The characteristic of the algorithm is that the parameters are automatically paired and the computational complexity is low. Simulation results show the effectiveness of the proposed algorithm.
\end{abstract}

\section{Introduction}

Source localization is one of the most important research fields of array signals. It has been widely used in radar, seismic exploration, sonar and many other fields. According to the distance between the source and the receiving array, the source location is divided into far field source localization and near field source localization. For far field source localization, the distance between the source and the receiving array is very far away, so the wavefront of the source can be regarded as a plane wave, and the position information of the source can be determined by the direction angle. But in the near field source localization, between the source and the receiver array distance, the source of the wavefront for spherical wave, the position information of the source to determine two parameters of direction angle and distance, so the near field source localization problem than the problem of far field source localization is more complicated, The existing far field source localization algorithms can not be directly applied to near field source localization, and new algorithms need to be developed. After more than twenty years of development, many scholars at home and abroad have studied the problem of source localization in the near field, and proposed many excellent algorithms ${ }^{[1-7]}$.

Swindlehurst et al proposed a near field source localization algorithm ${ }^{[3]}$ based on spatial Wigner distribution, but its estimation performance was poor; Challa $\mathrm{R} \mathrm{N}$ et al. Proposed a joint estimation algorithm $^{[4]}$ four order cumulant and the total least squares method of near field source and distance parameters based on two-dimensional DOA algorithm, by eigenvalue decomposition directly estimate the phase parameters, without spectral peak searching, but the parameter matching program, introduces additional error; Starer et al proposed a near field source localization method based on path tracking algorithmg $^{[5]}$, which uses a one-dimensional spectral peak search instead of two-dimensional peak search, and reduces the computational complexity of the MUSIC algorithm; J-H, Lee et al proposed an improved path tracking algorithm ${ }^{[6]}$ using known geometric paths. Compared with ${ }^{[5]}$, it reduces the computational complexity, but still needs one-dimensional peak search. Liang Junli et al proposed near field source localization algorithm ${ }^{[7]}$ based on parallel factor analysis, parallel factor model is extended to the high order cumulant domain, and three phase parameters by three-dimensional linear alternating least squares source, due to the need to construct a plurality of four order cumulant matrix, and the subspace decomposition, so the amount of calculation great. J-H, Lee et al proposed an improved path tracking algorithm ${ }^{[6]}$ using known geometric paths. Compared with ${ }^{[5]}$, it reduces the computational complexity, but still needs one-dimensional peak search.

Proposed a 2-D parameters estimation algorithm for near-field sources based on polarization sensitive array. The algorithm adopted the centro-symmetric uniform linear array, constructed three covariance matrices by the particular array labeling, and taken advantage of the total least squares and 
subspace decomposition method to estimate the bearing and range parameters. The characteristic of the algorithm is that the parameters are automatically paired and the computational complexity is low. It can be applied to additive Gauss white noise environment.

\section{Receiver array and signal model}

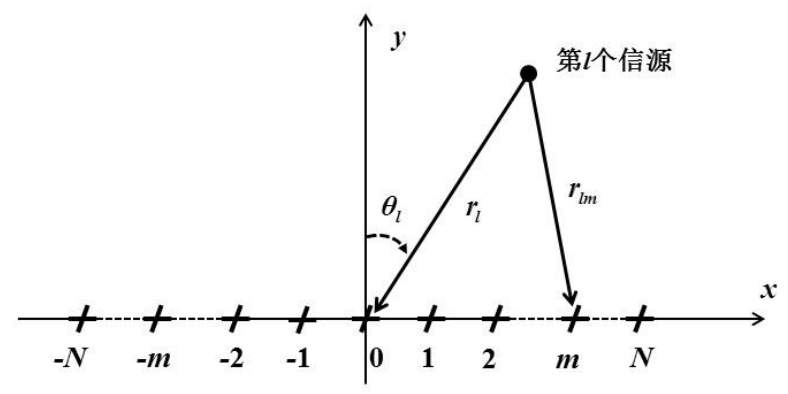

Figure 1. Finite Polarization Sensitive Array Model

The receiving array as shown in Figure 1, the receiver array is the extension of $\mathrm{X}$ axis symmetrical uniform linear array, to coordinate origin as the center, consists of a $2 \mathrm{~N}+1$ and the axis direction of the dipole on a sensor spacing of $\mathrm{D}$, electric field are induced from the $\mathrm{X}$ and $\mathrm{Y}$ directions. $\mathrm{A} \mathrm{L}(\mathrm{L}<\mathrm{N}-1)$ non Gauss and uncorrelated near field narrowband source is incident on the receiving array, and the coordinate origin $(0,0)$ is chosen as the phase reference element, and the direction angle and distance of the $\mathrm{L}$ source are represented as theta $\mathrm{L}, \mathrm{rl}$. Assuming that the noise on the array is additive Gauss white noise, then the output of the first pair of dipole pairs, the $\mathrm{X}$ direction and the $\mathrm{Y}$ direction can be represented as $\mathrm{m}$ :

$$
\begin{aligned}
& x_{m}{ }^{[x]}(t)=\sum_{l=1}^{L} s_{l}(t) e^{j\left(\omega_{x l} m+\phi_{x l} m^{2}\right)} u_{l}^{[x]}+n_{m}{ }^{[x]}(t) \\
& x_{m}{ }^{[y]}(t)=\sum_{l=1}^{L} s_{l}(t) e^{j\left(\omega_{x l} m+\phi_{x l} m^{2}\right)} u_{l}^{[y]}+n_{m}{ }^{[y]}(t)
\end{aligned}
$$

Type

$$
\begin{gathered}
\omega_{l}=-2 \pi \frac{d}{\lambda_{l}} \sin \theta_{l} \\
\phi_{l}=\pi \frac{d^{2}}{\lambda_{l} r_{l}} \cos ^{2} \theta_{l}
\end{gathered}
$$

$\mathrm{UL}$ is the output coefficient vector of the $\mathrm{X}$ axis and the $\mathrm{Y}$ axis, assuming that the dipoles are consistent with each other:

$$
u_{l}=\left[\begin{array}{l}
u_{l}^{[x]} \\
u_{l}^{[y]}
\end{array}\right]=\left[\begin{array}{c}
-\cos \gamma_{l} \\
j \cos \theta_{l} \sin \gamma_{l}
\end{array}\right]
$$

In the formula, $\gamma_{l}$ is the phase descriptor of the polarization state of the electromagnetic wave, that is, the electric field amplitude ratio between the $\mathrm{Y}$ direction and the $\mathrm{X}$ direction, referred to as the polarization auxiliary angle. $\gamma \in[0, \pi / 2]$ 。

In this case, the following assumptions are made:

Hypothesis 1: the source is a non Gauss, uncorrelated stationary random process;

Hypothesis 2: noise is additive Gauss white noise, and uncorrelated with the source;

Suppose 3: the array spacing is the minimum wavelength of the received signal. 


\section{Algorithm description}

Using the signals received by sensors with different array labels, the following correlation matrices are constructed

$$
\begin{aligned}
& \mathbf{R}_{1}(m, n)=\left\{E\left(\left(x_{n-m}{ }^{[x]}(t)\right)\left(x_{n-m-1}{ }^{[x]}(t)\right)^{\mathrm{H}}\right)\right\}^{*} \\
& =\left\{E \left[\left(\sum_{l=1}^{L} s_{l}(t) e^{j\left(\omega_{l}(n-m)+\phi_{l}(n-m)^{2}\right)} \boldsymbol{u}_{l}^{[x]}+n_{n-m}^{[x]}(t)\right)\right.\right. \\
& \left.\left.\left(\sum_{l=1}^{L} s_{l}(t) e^{j\left(\omega_{l}(n-m-1)+\phi_{l}(n-m-1)^{2}\right)} u_{l}^{[x]}+n_{n-m-1}^{[x]}(t)\right)^{\mathrm{H}}\right]\right\}^{*} \\
& =\sum_{l=1}^{L} r_{s l}\left|u_{l}^{[x]}\right|^{2} e^{j\left(\omega_{l}-\phi_{l}\right)} e^{j 2 \phi_{l}(n-m)}
\end{aligned}
$$

Matrix form

$\mathbf{R}_{1}(m, n)=\mathbf{A}\left(\phi_{l}\right) \mathbf{R}_{\mathbf{s}} \boldsymbol{\Omega} \boldsymbol{\Phi}^{*} \mathbf{A}^{\mathrm{H}}\left(\phi_{l}\right)$

Type

$$
\begin{aligned}
& \mathbf{A}\left(\phi_{l}\right)=\left[\boldsymbol{a}_{1}, \boldsymbol{a}_{2}, \cdots, \boldsymbol{a}_{\boldsymbol{L}}\right] \\
& \boldsymbol{a}_{\boldsymbol{l}}=\left[1, e^{j 2 \phi_{l}}, \cdots, e^{j 2 N \phi_{l}}\right]^{\mathrm{T}} \\
& \mathbf{R}_{\mathbf{s}}=\operatorname{diag}\left(r_{s 1}\left|u_{1}^{[x]}\right|^{2}, r_{s 2}\left|u_{2}^{[x]}\right|^{2}, \cdots, r_{s L}\left|u_{L}^{[x]}\right|^{2}\right) \\
& \mathbf{\Omega}_{x}=\operatorname{diag}\left(e^{j \omega_{l}}, e^{j \omega_{2}}, \cdots, e^{j \omega_{L}}\right) \\
& \mathbf{\Phi}_{y}=\operatorname{diag}\left(e^{j \phi_{x l}}, e^{j \phi_{x 2}}, \cdots, e^{j \phi_{x L}}\right)
\end{aligned}
$$

Similarly, the following two order two statistic matrices are constructed

$$
\begin{aligned}
\mathbf{R}_{2}(m, n) & =E\left(\left(x_{m-n+1}{ }^{[x]}(t)\right)\left(x_{m-n}{ }^{[x]}(t)\right)^{\mathrm{H}}\right) \\
& =\mathbf{A}\left(\phi_{l}\right) \mathbf{R}_{\mathbf{S}} \boldsymbol{\Omega} \boldsymbol{\Phi} \mathbf{A}^{\mathrm{H}}\left(\phi_{l}\right) \\
\mathbf{R}_{3}(m, n) & =\left(\mathbf{R}_{2}(m, n)\right)^{\mathrm{H}} \\
& =\mathbf{A}\left(\phi_{l}\right) \mathbf{R}_{\mathbf{S}} \boldsymbol{\Omega}^{*} \boldsymbol{\Phi}^{*} \mathbf{A}^{\mathrm{H}}\left(\phi_{l}\right)
\end{aligned}
$$

Construction matrix

$$
\mathbf{R}=\left[\begin{array}{l}
\mathbf{R}_{1} \\
\mathbf{R}_{2} \\
\mathbf{R}_{3}
\end{array}\right]=\left[\begin{array}{l}
\mathbf{B} \\
\mathbf{B} \boldsymbol{\Omega}^{2} \\
\mathbf{B} \boldsymbol{\Phi}^{* 2}
\end{array}\right] \mathbf{A}^{*}\left(\phi_{l}\right)
$$

Among

$$
\mathbf{B}=\mathbf{A}\left(\phi_{l}\right) \mathbf{R}_{\mathbf{S}} \boldsymbol{\Omega} \boldsymbol{\Phi}^{*}
$$


Singular value decomposition of matrix, $\mathbf{R}=\mathbf{U S V}^{\mathrm{H}}$, The singular values are sorted from large to small, and the left singular vectors corresponding to the maximum singular values of $\mathrm{L}$ are extracted, Structured signal subspace, $\mathbf{E}_{\mathrm{S}}=\left[e_{1}, \cdots, e_{L}\right]$, And divide it into three dimensional matrices $\mathbf{E}_{\mathbf{0}}, \mathbf{E}_{\mathbf{1}}, \mathbf{E}_{\mathbf{2}}$, as a result of

$$
R\left\{\mathbf{E}_{\mathrm{S}}\right\}=R\{\mathbf{B}\}=R\left\{\mathbf{E}_{0}\right\}=R\left\{\mathbf{E}_{1}\right\}=R\left\{\mathbf{E}_{2}\right\}
$$

Therefore, there must be a non singular $L \times L$ matrix $\mathbf{T}$,

$$
\mathbf{E}_{S} \mathbf{T}=\left[\begin{array}{l}
\mathbf{E}_{\mathbf{0}} \\
\mathbf{E}_{\mathbf{1}} \\
\mathbf{E}_{\mathbf{2}}
\end{array}\right] \mathbf{T}=\left[\begin{array}{l}
\mathbf{B} \\
\mathbf{B} \boldsymbol{\Omega}^{2} \\
\mathbf{B} \boldsymbol{\Phi}^{* 2}
\end{array}\right]
$$

That is

$$
\begin{aligned}
& \mathbf{E}_{\mathbf{0}} \mathbf{T}=\mathbf{B} \quad \mathbf{E}_{\mathbf{1}} \mathbf{T}=\mathbf{B} \boldsymbol{\Omega}^{2} \quad \mathbf{E}_{\mathbf{2}} \mathbf{T}=\mathbf{B} \boldsymbol{\Phi}^{* 2} \\
& \begin{array}{|l}
\mathbf{E}_{\mathbf{1}}=\mathbf{E}_{\mathbf{0}} \boldsymbol{\Psi}_{1} \\
\text { Among }
\end{array}
\end{aligned}
$$

$$
\boldsymbol{\Psi}_{1}=\mathbf{T} \boldsymbol{\Omega}^{2} \mathbf{T}^{-1} \quad \boldsymbol{\Psi}_{2}=\mathbf{T} \boldsymbol{\Phi}^{* 2} \mathbf{T}^{-1}
$$

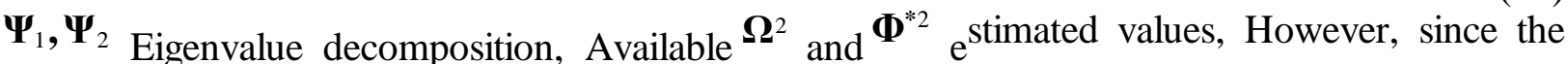
eigenvalues of the two matrices are independent, the order of their eigenvectors may be different, and the order of eigenvalues is not one-to-one, Therefore, the introduction of parametric pairing procedures is well known, and parameter matching procedures introduce additional errors, so this section uses the method in document [9], $\boldsymbol{\Psi}_{1}, \boldsymbol{\Psi}_{2}$ Eigenvalue decomposition, the method does not require eigenvalue decomposition, and can directly estimate the closed form solution of the source phase parameters.

\section{$\boldsymbol{\Psi}_{1}$ Eigenvalue decomposition}

$$
\mathbf{V}_{1} \mathbf{U}_{1}=\Psi_{1} \mathbf{U}_{1}
$$

Among

$$
\begin{aligned}
& \mathbf{U}_{1}=\left[\boldsymbol{u}_{1}, \boldsymbol{u}_{2}, \cdots, \boldsymbol{u}_{L}\right]^{\mathrm{T}} \\
& \mathbf{V}_{1}=\operatorname{diag}\left(\boldsymbol{v}_{1}, \boldsymbol{v}_{2}, \cdots, \boldsymbol{v}_{L}\right)
\end{aligned}
$$

By formula (21), $\boldsymbol{\Psi}_{1}, \boldsymbol{\Psi}_{2}$ having the same characteristic vector and different eigenvalues, That is $\mathbf{U}_{1}$, the characteristic vector $\boldsymbol{\Psi}_{2}$, Set $\mathbf{V}_{2}$ as the eigenvalue matrix of the matrix $\boldsymbol{\Psi}_{2}$, so there is

$$
\mathbf{V}_{2} \mathbf{U}_{1}=\Psi_{2} \mathbf{U}_{1}
$$

set up

$$
\boldsymbol{\Psi}_{2} \mathbf{U}_{1}=\left[\zeta_{21}, \zeta_{22}, \cdots, \zeta_{2 L}\right]
$$




$$
\mathbf{V}_{2}=\operatorname{diag}\left(v_{21}, v_{22}, \cdots, v_{2 L}\right)
$$

The eigenvalues $\boldsymbol{\Psi}_{2}$ that can be computed according to formula (27), among $j=1,2, \cdots, L$

$$
v_{2 j}=\frac{1}{L} \sum_{l=1}^{L} \frac{\zeta_{2 l}}{u_{j l}}
$$

Then, according to the formula $(11) \sim(12)$, the estimation of the phase parameters of the near field source can be derived $\omega_{x}, \phi_{x}$

$$
\begin{gathered}
\omega_{l}=0.5 \operatorname{angle}\left(\mathbf{V}_{1}\right) \\
\phi_{l}=-0.5 \operatorname{angle}\left(\mathbf{V}_{2}\right)
\end{gathered}
$$

Then, according to formula (3) (4), the two-dimensional parameter estimation of polarization array near field source can be obtained:

$$
\begin{aligned}
& \theta_{l}=-\operatorname{asin}\left(\frac{\omega_{l} \lambda}{2 \pi d}\right) \\
& \hat{r}_{l}=\frac{\pi d^{2}}{\lambda \phi_{l}} \cos \left(\theta_{l}\right)
\end{aligned}
$$

\section{Simulation experiment}

Two equal amplitude modulated amplitude modulation sources S1 and S2 are used in this experiment, Carrier frequency, respectively $f_{1}=4 \mathrm{MHz}, f_{2}=2 \mathrm{MHz}$, Sampling frequency is $16 \mathrm{MHz}$, The direction angles are $\theta_{1}=20^{\circ}, \theta_{2}=30^{\circ}$, distance parameter is $\mathrm{r} 1=0.2, \mathrm{r} 2=0.4$. The estimated values of the direction and distance parameters of the source are the degree and the wavelength respectively. In the experiment, the mean square error criterion (MSE) is adopted as the performance evaluation criterion

$$
M S E=10 \log _{10}\left(\frac{1}{N_{r} p^{2}} \sum_{r=1}^{N_{r}}(p-p)^{2}\right)
$$

$p$ to estimate parameters, $N_{r}$ is the number of experiments for Monte-Carlo.

The method proposed in this section uses a centrosymmetric homogeneous linear array, as shown in Figure 1, consisting of 9 polarized dipole pairs, with the element spacing being 1/4 of the shortest wavelength. The noise on the element is additive Gauss white noise. To demonstrate the effectiveness of the proposed algorithm, the proposed algorithm is compared with the algorithm in the literature [8] (applied to a uniform linear array).

Experiment 1: The signal-to-noise ratio of the experiment is $10 \mathrm{~dB}$, and the number of sampling points varies from 256 to 1536, each sampling points for the 200 Monte-Carlo experiment, two source $\mathrm{S} 1$ and S2 direction angle and distance curve parameter estimation mean square error value varies with the number of snapshots is shown in Fig. 2 Fig. 3. 

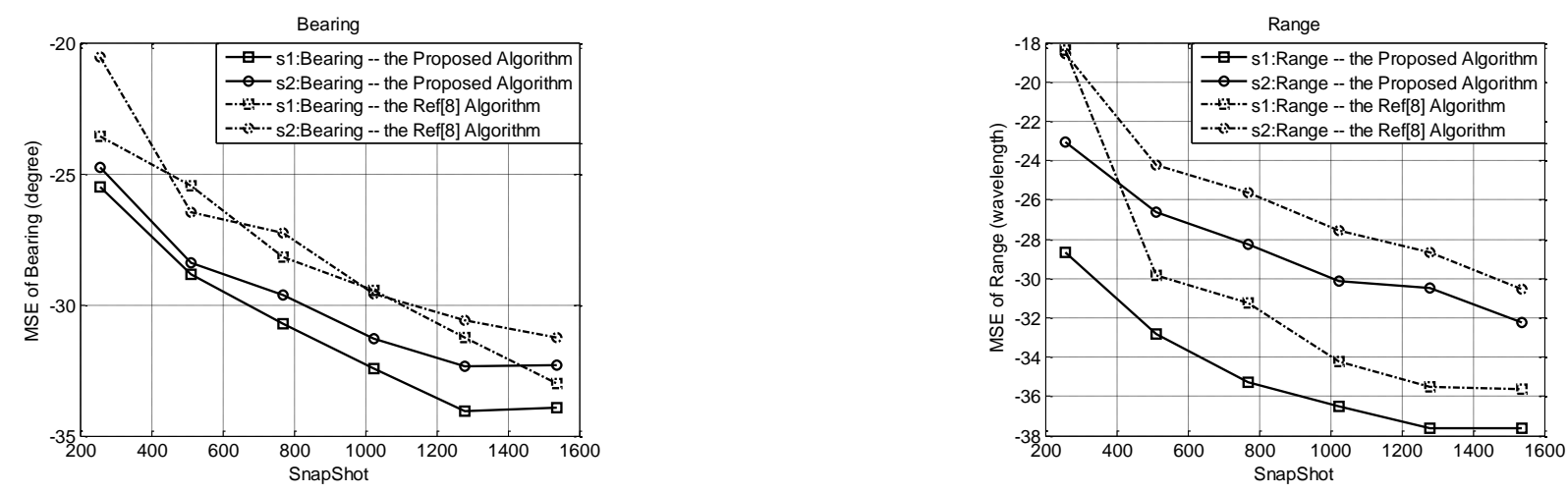

Figure 2. Finite MSE curve along with Snapshot of DOA Figure 3. Finite MSE curve along with Snapshot of Range

By $2 \sim$ in Figure 3 shows, this section presents the algorithm can accurately estimate the parameters of two-dimensional source, direction angle and distance parameters of mean square error decreased with increasing number of snapshots, and has higher estimation accuracy and better estimation performance than the [8] algorithm.

Experiment 2: The number of snapshots is 512 to $30 \mathrm{~dB}$ than the $5 \mathrm{~dB}$ change in signal-to-noise, each signal-to-noise compared to 200 Monte-Carlo experiments, two source S1 and S2 direction angle and distance parameter estimation mean square error value with the SNR curve as shown in Fig. $4 \sim$ is shown in Fig. 5.
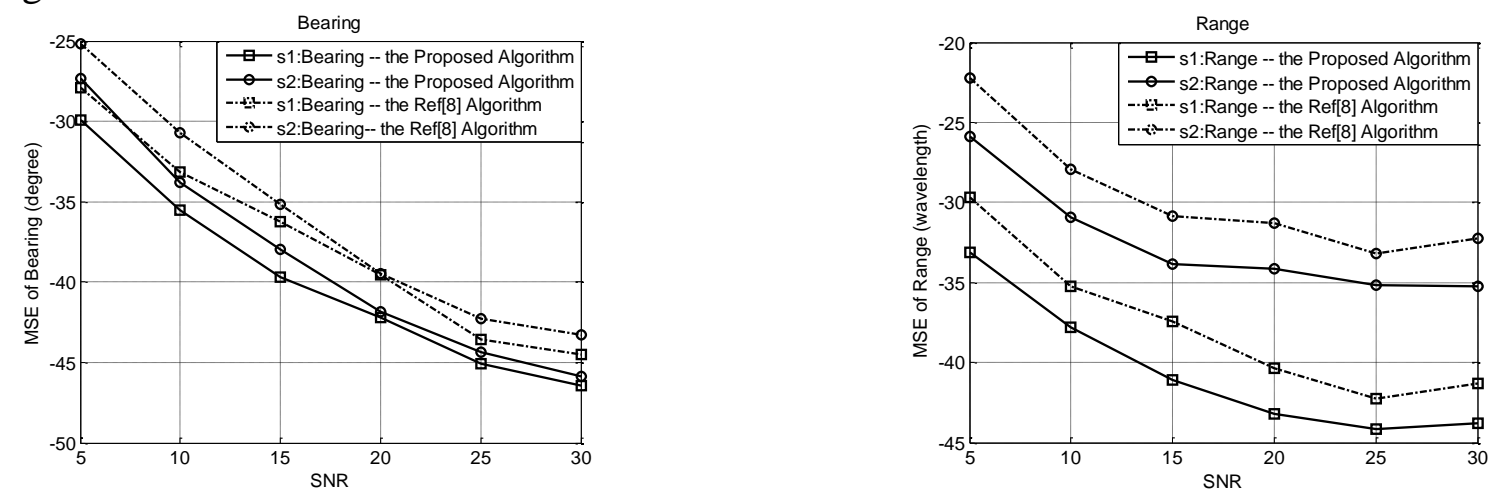

Figure 4. Finite MSE curve along with SNR of DO Figure 5. Finite MSE curve along with SNR of Range

By $4 \sim$ in Figure 5 shows, this section presents the algorithm can accurately estimate the parameters of two-dimensional source, direction angle and distance parameters of mean square error decreases with the increase of SNR and better estimation performance than [8] algorithm in the literature.

From Experiment 1 and 2 simulation results shows that both the number of snapshots in the experiment, or the signal-to-noise ratio in the experiment, the method presented in this section are able to accurately estimate the parameters of two-dimensional source, in the distance parameter estimation, estimation performance of two kinds of methods of CITIC S1 source are slightly better than the source $\mathrm{S} 2$, when the distance is near the source array, the distance estimation performance is relatively good, the conclusion and references [10] theoretical results.

\section{Conclusion}

Proposed a 2-D parameters estimation algorithm for near-field sources based on polarization sensitive array. The algorithm adopted the centro-symmetric uniform linear array, constructed three covariance matrices by the particular array labeling, and taken advantage of the total least squares and subspace decomposition method to estimate the bearing and range parameters. The characteristic of the algorithm 
is that the parameters are automatically paired and the computational complexity is low. The algorithm has the advantage of parameter matching, without additional parameter matching procedures, improve the estimation performance of the algorithm, due to the use of two order statistics, this paper estimates the algorithm only adapts to the additive Gauss white noise environment, simulation results show the effectiveness of the proposed algorithm.

\section{Acknowledgements}

This work is supported by the Nature Science Foundation of Jilin Province (Grant 20140101177JC), the Science and Technology Research Project of Department of Education of Jilin Province (Grant 2016057) and the Distinguished Young Scholars Foundation of Jilin City (Grant 201467007)

\section{Literature References}

[1] N. Tayem, K. Majeed and A. A. Hussain. Two-Dimensional DOA Estimation Using Cross-Correlation Matrix With L-Shaped Array[J]. IEEE Antennas and Wireless Propagation Letters, 2016, 15(1): 1077-1080.

[2] H. Jiang, J. K. Zhang and K. M. Wong. Joint DOD and DOA Estimation for Bistatic MIMO Radar in Unknown Correlated Noise[J]. IEEE Transactions on Vehicular Technology, 2015, 64(11): 5113-5125.

[3] A.L.Swindlehurst and T.Kailath. Passive direction-of-arrival and range estimation for near-field sources[C]. IEEE Spectrum Estimation and Modeling. Workshop, Minneapolis, MN USA, 1988, 123-128.

[4] Raghu N Challa and Sanyogita Shamsunder. High-order subspace-based algorithms for passive localization of near-field sources[C]. 29th Asilomar Conference, November.1995, pp.777-781.

[5]D.Starer and A.Nehorai. Passive localization of near-field sources by path following[J]. IEEE Trans, SP, March, 1994, 42(3):677-680.

[6] J-H Lee, C-M Lee and K-K Lee. A modified path-following algorithm using a known algebraic path[J]. IEEE Trans, SP, May, 1999, 47(5):1407-1409.

[7] Abred-Meraim K and Hua Y. 3-D near field source localization using second order statistics[C]. Conference Record of the Thirty-First Asilomar Conference on Signals, Systems \& Computers, Pacific Grove, CA USA, 1997, 1307-1311.

[8] Yuen N and Friedlander B. Performance analysis of higher order ESPRIT for localization of near-field sources[J]. IEEE Trans on SP, 1998, 46(3):709-719.

[9] Jun Liu,Zheng Liu and Qin Liu:Direction and polarization estimation for coherent sources using vector sensors,Journal of Systems Engineering and Electronics. 2013(04)

[10] LU Z,ZOUBIR A M: Generalized bayesian information criterion for source enumeration in array processing,IEEE Transactions on Signal Processing.(2013) 